\title{
An insight into structure and stability of DNA in ionic liquids from molecular dynamics simulation and experimental studies
}

\begin{abstract}
Molecular dynamics simulation and biophysical analysis were employed to reveal the characteristics and the influence of ionic liquids (ILs) on the structural properties of DNA. Both computational and experimental evidence indicate that DNA retains its native Bconformation in ILs. Simulation data show that the hydration shells around the DNA phosphate group were the main criteria for DNA stabilization in this ionic media. Stronger hydration shells reduce the binding ability of ILs' cations to the DNA phosphate group, thus destabilizing the DNA. The simulation results also indicated that the DNA structure maintains its duplex conformation when solvated by ILs at different temperatures up to $373.15 \mathrm{~K}$. The result further suggests that the thermal stability of DNA at high temperatures is related to the solvent thermodynamics, especially entropy and enthalpy of water. All the molecular simulation results were consistent with the experimental findings. The understanding of the properties of IL-DNA could be used as a basis for future development of specific ILs for nucleic acid technology.
\end{abstract}

Keyword: Ionic liquids (ILs); DNA 